\title{
New endoscopic over-the-scope clip system for closure of a chronic tracheoesophageal fistula
}

Acquired tracheoesophageal fistula is a rare complication caused by a variety of conditions. Along with trauma and malignancy, cuff-related tracheal injury has become the most common etiology. As few cases have been reported, with different clinical presentations and solutions for treatment, the results and conclusions are difficult to interpret $[1-3]$. This report describes a new, practical approach to the treatment of large tracheoesophageal fistulas.

In September 2008, a 74-year-old woman was admitted to another facility because of interstitial pneumonia, eventually requiring intubation and tracheostomy. After 1 month of mechanically assisted ventilation, the patient was discharged. Within 2 months the patient developed recurrent bronchitis and coughing after swallowing, requiring a bronchoscopy, which revealed the presence of chronic tracheoesophageal fistula. Enteral nutrition was commenced through a percutaneous endoscopic gastrostomy.

In March 2009, the patient was transferred to our institute. A computed tomography (CT) scan, Gastrografin esophagography, esophagogastroduodenoscopy (EGDS), and bronchoscopy confirmed the presence of the chronic tracheoesophageal fistula. Bronchoscopy showed 40\% tracheal stenosis, $4 \mathrm{~cm}$ below the vocal chords, and a fistula $1 \mathrm{~cm}$ below the stenosis. EGDS showed the fistula to be at a distance of $20 \mathrm{~cm}$ from the mouth ( $\bullet$ Fig. 1). We decided to treat the tracheoesophageal fistula with the over-thescope clip (OTSC; Ovesco Endoscopy $\mathrm{GmbH}$, Tübingen, Germany) system. The OTSC system is a new endoscopic tool for compression of large tissue areas, and is indicated for difficult-to-control nonvaricose bleeding, and lesions or perforations of the gastrointestinal tract [4].

Under endoscopic and radiologic control, we placed a $10-\mathrm{mm}$ wide OTSC to close the fistula ( Figs. 2,3 ). The patient's fever resolved and there was improvement in her respiratory condition. Full closure of the fistula was seen on EGDS, bronchoscopy, and Gastrografin esophagography carried out at 2 and 4 weeks of follow-up (๑ Figs. 3, 4). No clinical complications occurred, and the patient started eating normally through the mouth.

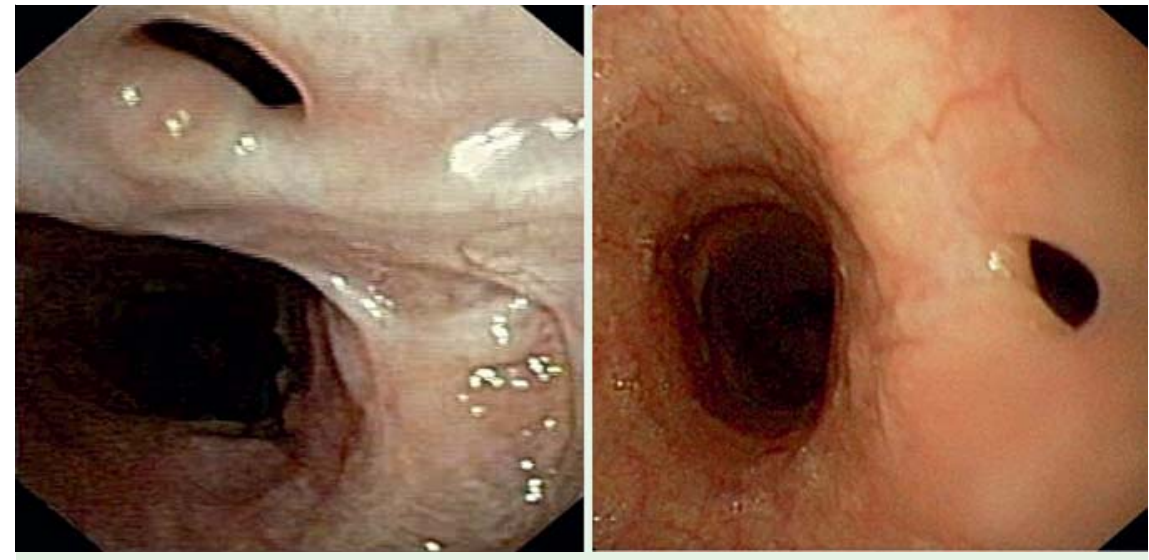

Fig. 1 Bronchoscopy (left panel) and esophagogastroduodenoscopy (right panel) showing a tracheoesophageal fistula in a 74-year-old woman.
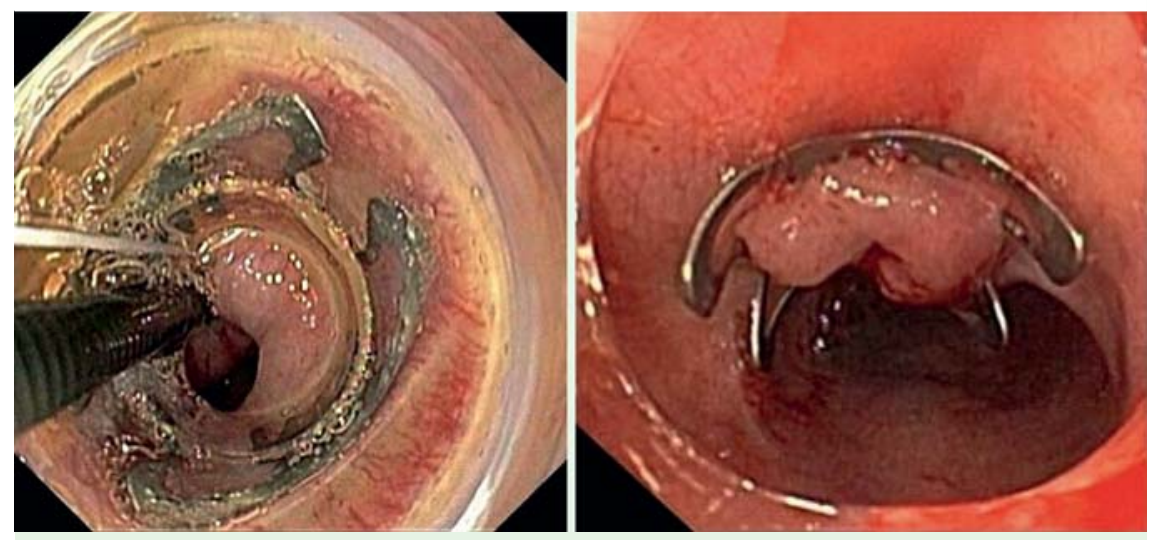

Fig. 2 Endoscopic view of closure of the fistula with a 10-mm wide over-the-scope clip.
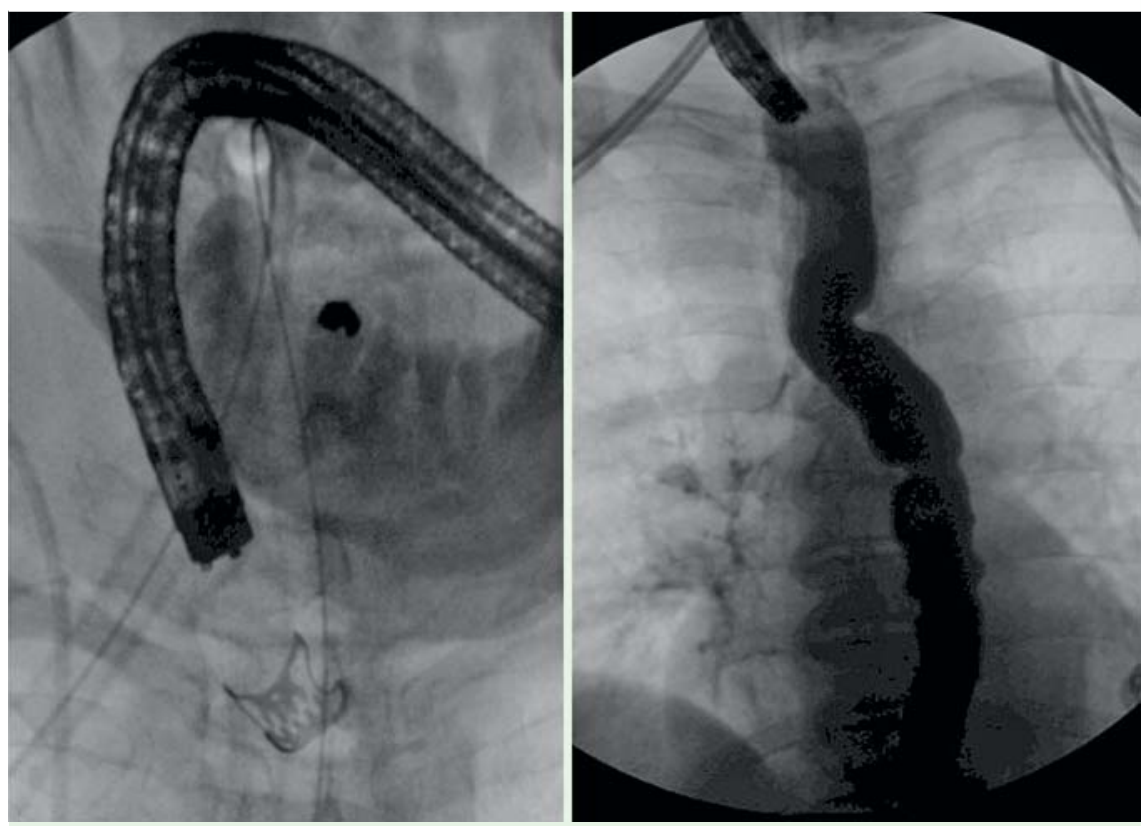

Fig. 3 Radiographs showing the over-the-scope clip at the site of the fistula during the procedure (left panel), with closure of the fistula seen at follow-up (right panel). 

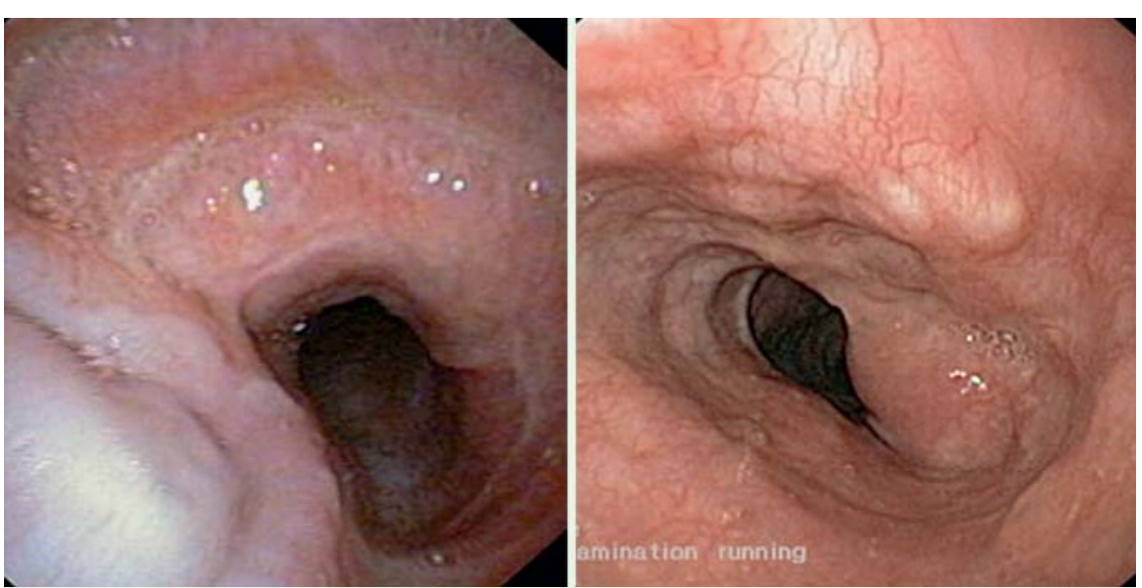

Fig. 4 Resolution of the tracheoesophageal fistula seen on bronchoscopy (left panel) and esophagogastroduodenoscopy (right panel).

Endoscopy_UCTN_Code_TTT_1AO_2AI

M. Traina, G. Curcio, I. Tarantino, S. Soresi, L. Barresi, P. Vitulo, B. Gridelli IsMeTT, UPMC, Palermo, Italy
3 Qadeer MA, Dumot JA, Vargo IJ et al. Endoscopic clips for closing esophageal perforations: case report and pooled analysis. Gastrointest Endosc 2007; 66: 605-611

4 Kirschniak A, Kratt T, Stüker D et al. A new endoscopic over-the-scope clip system for treatment of lesions and bleeding in the $\mathrm{Gl}$ tract: first clinical experiences. Gastrointest Endosc 2007; 66: 162 - 167

Bibliography

DOI $10.1055 / \mathrm{s}-0029-1243824$

Endoscopy 2010; 42: E54 - E55

(c) Georg Thieme Verlag KG Stuttgart · New York . ISSN 0013-726X

\section{Corresponding author}

\section{G. Curcio, MD}

Department of Gastroenterology

IsMeTT, UPMC

Via Tricomi 1

Palermo 90100

Italy

Fax: +39-091-2192400

gcurcio@ismett.edu 